\title{
Simulations of scraping on the AGS beam dump
}

\author{
C.J. Gardner
}

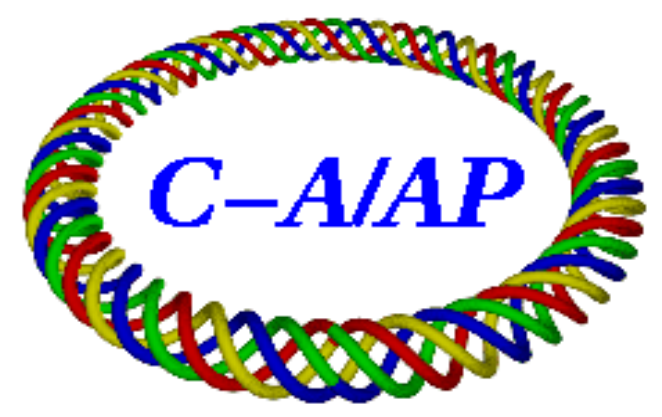

\section{Collider-Accelerator Department Brookhaven National Laboratory Upton, NY 11973}

Notice: This document has been authorized by employees of Brookhaven Science Associates, LLC under Contract No. DE-AC02-98CH10886 with the U.S. Department of Energy. The United States Government retains a nonexclusive, paid-up, irrevocable, world-wide license to publish or reproduce the published form of this document, or allow others to do so, for United States Government purposes. 


\title{
Simulation of Scraping on the AGS Beam Dump
}

\author{
C.J. Gardner
}

October 28, 2013

The AGS beam dump is described in reference [1]. Because there is no fast dipole magnet to kick the beam into the dump, the dump is used as a beam scraper into which circulating beam is moved over a period of many turns around the machine. To get a better understanding of what happens during scraping, a relatively simple model and computer code have been developed to simulate the process. We report here on the simulation results for protons at the nominal extraction kinetic energy of $22.874 \mathrm{GeV}$ and for gold ions $(\mathrm{Au} 77+)$ at the nominal extraction kinetic energy of 8.86 $\mathrm{GeV}$ per nucleon.

In Sections 1 and $\mathbf{2}$ the optimum position and slope of the scraper are determined. The circulating beam distribution at the start of scraping is defined in Section 3. Particle tracking and energy loss in the scraper material (copper) are discussed in Sections 4 and 5. Turn-by-turn tracking and the determination of the maximum energy loss for which particles emerging from the scraper will survive subsequent turns around the machine are discussed in Sections 6 and 7. Tracking results for the first hits of particles on the scraper are given in Section 8. The results for the fraction of Au77+ ions lost in and downstream of the scraper are given in Section 9. Tracking results for the second hits of protons on the scraper are given in Section 10. Inelastic nuclear interactions of protons with the scraper material are discussed in Section 11. The results for the fraction of protons lost in and downstream of the scraper are given in Section 12 . The effect of multiple coulomb scattering is discussed in Section 13.

The main results of the report are those given in Sections 9 and $\mathbf{1 2}$. 


\section{Scraper Position and Beam Envelope}

We assume that the scraper is positioned in a drift section so that as the circulating beam envelope is moved radially inward, the envelope touches the upstream end of the scraper first. We use coordinate $S$ to denote the distance along the drift with $S=0$ at the upstream end of the scraper. At the downstream end $S$ is equal to

$$
\underline{L=2.022348 \text { meters. }} \text {. }
$$

We take the horizontal (i.e. radial) position of the scraper aperture at $S=0$ to be

$$
\underline{H_{0}=-54.1825 \mathrm{~mm}} .
$$

The MAD model of the AGS shows that this then becomes a limiting aperture of the machine.

Let $\alpha_{0}, \beta_{0}$, and $\gamma_{0}$ be the horizontal Courant-Snyder parameters of the lattice at $S=0$. Then, with the circulating beam envelope just touching the scraper at $S=0$, the position of the equilibrium orbit (at $S=0$ ) is

$$
d_{0}=H_{0}+\sqrt{\epsilon \beta_{0}}
$$

where $\pi \epsilon$ is the circulating beam emittance. The MAD model of the AGS gives

$$
\underline{\beta_{0}=17.3224 \text { meters. }} .
$$

At a distance $S$ along the drift, the position of the equilibrium orbit is

$$
d=d_{0}+d_{0}^{\prime} S
$$

where $d_{0}^{\prime}$ is the angle of the orbit at $S=0$. The lattice parameters at distance $S$ are

$$
\begin{gathered}
\beta=\beta_{0}-2 \alpha_{0} S+\gamma_{0} S^{2} \\
\alpha=\alpha_{0}-\gamma_{0} S \\
\gamma=\gamma_{0}
\end{gathered}
$$

where

$$
\beta_{0} \gamma_{0}-\alpha_{0}^{2}=1
$$

The radially inward position of the beam envelope at distance $S$ is then

$$
E=d-\sqrt{\epsilon \beta}
$$


and using (3) and (5) we have

$$
E=H_{0}+\sqrt{\epsilon \beta_{0}}+d_{0}^{\prime} S-\sqrt{\epsilon \beta} .
$$

Here it is convenient to introduce parameter

$$
\mathcal{A}(S)=\frac{1}{S}\left(\beta_{0}-\sqrt{\beta \beta_{0}}\right)
$$

where

$$
\beta \beta_{0}=\left(\beta_{0}-\alpha_{0} S\right)^{2}+S^{2} .
$$

We then have

$$
\sqrt{\epsilon \beta_{0}}-\sqrt{\epsilon \beta}=\frac{S \mathcal{A}}{\beta_{0}} \sqrt{\epsilon \beta_{0}}
$$

and

$$
E=H_{0}+\left\{d_{0}^{\prime}+\frac{\mathcal{A}}{\beta_{0}} \sqrt{\epsilon \beta_{0}}\right\} S
$$

For $S \ll \beta_{0}$ we have

$$
\begin{gathered}
\sqrt{\beta \beta_{0}}=\beta_{0}-\alpha_{0} S+\frac{S^{2}}{2 \beta_{0}}+\frac{\alpha_{0} S^{3}}{2 \beta_{0}^{2}}+\frac{S^{4}}{8 \beta_{0}^{3}}\left(4 \alpha_{0}^{2}-1\right)+\cdots \\
\mathcal{A}(S)=\alpha_{0}-\frac{1}{2} \frac{S}{\beta_{0}}-\frac{\alpha_{0}}{2} \frac{S^{2}}{\beta_{0}^{2}}-\frac{1}{8}\left(4 \alpha_{0}^{2}-1\right) \frac{S^{3}}{\beta_{0}^{3}}+\cdots
\end{gathered}
$$

and therefore

$$
\mathcal{A}(0)=\alpha_{0} .
$$

The MAD model of the AGS gives

$$
\underline{\alpha_{0}=1.5268} .
$$

A plot of $\mathcal{A}(S)$ for $0 \leq S \leq L$ is shown in Figure 1. The function is monotonically decreasing with

$$
\mathcal{A}(L)=1.4561 .
$$

We assume that the equilibrium orbit angle at $S=0$ depends linearly on the orbit position. Thus we can write

$$
d_{0}^{\prime}=\mathcal{M}_{0}-\frac{k_{0}}{\beta_{0}} d_{0}
$$


where $\mathcal{M}_{0}$ and $k_{0}$ are constants. The MAD model of the orbit bump [2] at the scraper gives

$$
\underline{k_{0}=1.097} .
$$

The constant $\mathcal{M}_{0}$ is an independent parameter that can be adjusted as needed. Using (3) we then have

$$
\begin{gathered}
d_{0}^{\prime}=\mathcal{M}_{0}-\frac{k_{0}}{\beta_{0}}\left(H_{0}+\sqrt{\epsilon \beta_{0}}\right) \\
d_{0}^{\prime}+\frac{\mathcal{A}}{\beta_{0}} \sqrt{\epsilon \beta_{0}}=\mathcal{M}_{0}-\frac{k_{0} H_{0}}{\beta_{0}}+\left(\frac{\mathcal{A}}{\beta_{0}}-\frac{k_{0}}{\beta_{0}}\right) \sqrt{\epsilon \beta_{0}}
\end{gathered}
$$

and the beam envelope (15) becomes

$$
E=H_{0}+\left\{\mathcal{M}_{0}-\frac{k_{0} H_{0}}{\beta_{0}}+\left(\frac{\mathcal{A}}{\beta_{0}}-\frac{k_{0}}{\beta_{0}}\right) \sqrt{\epsilon \beta_{0}}\right\} S .
$$

\section{The Scraper Slope}

We assume that the position of the scraper aperture at distance $S$ along the drift section is

$$
H=H_{0}+\mathcal{M} S
$$

where $\mathcal{M}$ is the slope of the scraper aperture with respect to the vacuum chamber centerline.

We want to choose the slope so that for any $S>0$ we have

$$
H<E \text {. }
$$

This ensures that as the circulating beam envelope is moved inward, the envelope always touches the upstream end of the scraper first. Thus we want

$$
H_{0}+\mathcal{M} S<H_{0}+\left\{d_{0}^{\prime}+\frac{\mathcal{A}}{\beta_{0}} \sqrt{\epsilon \beta_{0}}\right\} S
$$

which gives

$$
\mathcal{M}<d_{0}^{\prime}+\frac{\mathcal{A}}{\beta_{0}} \sqrt{\epsilon \beta_{0}} .
$$

Using (24) this becomes

$$
\mathcal{M}<\mathcal{M}_{0}-\frac{k_{0} H_{0}}{\beta_{0}}+\left(\frac{\mathcal{A}}{\beta_{0}}-\frac{k_{0}}{\beta_{0}}\right) \sqrt{\epsilon \beta_{0}} .
$$


Figure 1 and equation (22) show that

$$
\mathcal{A}(S)-k_{0}>0
$$

and it follows that (30) will be satisfied for all $\sqrt{\epsilon \beta_{0}}>0$ if we take

$$
\mathcal{M}=\mathcal{M}_{0}-\frac{k_{0} H_{0}}{\beta_{0}} .
$$

We can then write the envelope (25) as

$$
E=H_{0}+\left\{\mathcal{M}+\left(\frac{\mathcal{A}}{\beta_{0}}-\frac{k_{0}}{\beta_{0}}\right) \sqrt{\epsilon \beta_{0}}\right\} S
$$

and using (26) we have

$$
E=H+\left\{\left(\frac{\mathcal{A}}{\beta_{0}}-\frac{k_{0}}{\beta_{0}}\right) \sqrt{\epsilon \beta_{0}}\right\} S .
$$

Note that if the scraper slope $\mathcal{M}$ is given, we can adjust the orbit angle parameter $\mathcal{M}_{0}$ so that (32) is satisfied. This means that rather than having to adjust the scraper slope, one can simply adjust the orbit angle $\mathcal{M}_{0}$. This would be a useful feature to have in the AGS where at present one does not have independent control of orbit position and angle at the scraper. We assume here that the orbit angle and scraper slope have been adjusted so that

$$
\mathcal{M}_{0}=0
$$

in (21) and (32). This gives

$$
\begin{aligned}
& d_{0}^{\prime}=-\frac{k_{0}}{\beta_{0}} d_{0} \\
& \mathcal{M}=-\frac{k_{0} H_{0}}{\beta_{0}}
\end{aligned}
$$

and

$$
H=H_{0}-\frac{k_{0} H_{0}}{\beta_{0}} S
$$

Figure 2 shows a plot of $E(S)$ and $H(S)$ for $0 \leq S \leq L$ and $\epsilon=1.0 \mathrm{~mm}$ milliradians. Here we see that the beam envelope touches the scraper aperture at the upstream end and is inside the aperture at all points downstream. Putting $S=L$ in (34) and using (4), (20) and (22) gives the maximum separation

$$
E(L)-H(L)=0.0419 \sqrt{\epsilon \beta_{0}}
$$

between envelope and aperture. Figure 3 shows the separation $E(S)-H(S)$ for the case in which $\epsilon=1.0 \mathrm{~mm}$ milliradians. 


\section{Beam Distribution at Start of Scraping}

At the start of scraping, the border of the beam distribution at the upstream end of the scraper is the ellipse

$$
\gamma_{0}\left(Z_{0}-d_{0}\right)^{2}+2 \alpha_{0}\left(Z_{0}-d_{0}\right)\left(Z_{0}^{\prime}-d_{0}^{\prime}\right)+\beta_{0}\left(Z_{0}^{\prime}-d_{0}^{\prime}\right)^{2}=\epsilon
$$

which is centered on the equilibrium orbit and matched to the machine lattice. Here $Z_{0}$ and $Z_{0}^{\prime}$ are the position and angle of a beam particle and $d_{0}$ and $d_{0}^{\prime}$ are the position and angle of the equilibrium orbit. We assume that the beam particles are uniformly distributed over the area of the ellipse. Figure 4 shows the distribution for $\epsilon=1.0 \mathrm{~mm}$ milliradians. The ellipse is uniformly covered with 20055 particles. The red line marks the scraper aperture $H_{0}$ and the blue line marks the scraper slope given by (37). The circulating beam moves into the scraper as the ellipse center $\left(d_{0}, d_{0}^{\prime}\right)$ moves along the brown line given by $(36)$.

\section{Particle Tracking in the Scraper Drift}

Consider a circulating beam particle launched with position $Z_{0}$ and angle $Z_{0}^{\prime}$ at the upstream end of the scraper. If the particle does not hit the scraper then its position $Z$ at a distance $S$ along the scraper drift is

$$
Z=Z_{0}+Z_{0}^{\prime} S
$$

and its angle is

$$
Z^{\prime}=\frac{d Z}{d S}=Z_{0}
$$

We shall assume that these equations hold even if the particle hits and passes through the scraper. This means that we are neglecting the changes in angle due to multiple coulomb scattering. The effects of including the angle changes will be discussed in Section 13. We will use the term "track" to refer to the trajectory a particle would follow in the scraper assuming no interaction with the scraper material.

If $Z_{0}^{\prime} \neq \mathcal{M}$ then the particle trajectory (41) will intersect the line

$$
H=H_{0}+\mathcal{M} S
$$

at a distance

$$
S_{e}=\frac{Z_{0}-H_{0}}{\mathcal{M}-Z_{0}^{\prime}}
$$


along the drift. There are then four cases to consider. Each of these corresponds to one of the quadrants defined by the red and blue lines in Figure 4.

1. For $Z_{0} \leq H_{0}$ and $Z_{0}^{\prime}>\mathcal{M}$ we have

$$
Z_{0}-H_{0} \leq 0, \quad \mathcal{M}-Z_{0}^{\prime}<0, \quad 0 \leq S_{e} .
$$

It follows that for $S>S_{e}$ we have

$$
\begin{gathered}
\left(\mathcal{M}-Z_{0}^{\prime}\right) S<\left(\mathcal{M}-Z_{0}^{\prime}\right) S_{e} \\
\left(\mathcal{M}-Z_{0}^{\prime}\right) S<Z_{0}-H_{0} \\
H_{0}+\mathcal{M} S<Z_{0}+Z_{0}^{\prime} S
\end{gathered}
$$

and the particle is outside the scraper.

Similarly, for $0<S<S_{e}$ we have

$$
\begin{gathered}
\left(\mathcal{M}-Z_{0}^{\prime}\right) S_{e}<\left(\mathcal{M}-Z_{0}^{\prime}\right) S \\
Z_{0}-H_{0}<\left(\mathcal{M}-Z_{0}^{\prime}\right) S \\
Z_{0}+Z_{0}^{\prime} S<H_{0}+\mathcal{M} S
\end{gathered}
$$

and the particle is inside the scraper. If $S_{e}<L$ then the length of the track in the scraper is

$$
L_{T}=S_{e} \sqrt{1+Z_{0}^{\prime 2}} .
$$

The depth of the track is defined to be

$$
D_{T}=\left(Z_{0}-H_{0}\right) / 2 .
$$

If $S_{e} \geq L$ then the length of the track is

$$
L_{T}=L \sqrt{1+Z_{0}^{\prime 2}}
$$

and the depth is

$$
D_{T}=\left(Z_{0}-H_{0}+Z_{L}-H_{L}\right) / 2
$$

where

$$
Z_{L}=Z_{0}+Z_{0}^{\prime} L
$$

and

$$
H_{L}=H_{0}+\mathcal{M} L
$$


2. For $Z_{0} \leq H_{0}$ and $Z_{0}^{\prime}<\mathcal{M}$ we have

$$
Z_{0}-H_{0} \leq 0, \quad 0<\mathcal{M}-Z_{0}^{\prime}, \quad S_{e} \leq 0 .
$$

It follows that for $0<S$ we have

$$
Z_{0}+Z_{0}^{\prime} S<H_{0}+\mathcal{M} S
$$

and the particle is inside the scraper. The length and depth of the track in the scraper are then given by (54) and (55).

3. For $Z_{0} \geq H_{0}$ and $Z_{0}^{\prime}<\mathcal{M}$ we have

$$
Z_{0}-H_{0} \geq 0, \quad \mathcal{M}-Z_{0}^{\prime}>0, \quad S_{e} \geq 0 .
$$

It follows that for $0<S<S_{e}$ we have

$$
\begin{gathered}
\left(\mathcal{M}-Z_{0}^{\prime}\right) S<\left(\mathcal{M}-Z_{0}^{\prime}\right) S_{e} \\
\left(\mathcal{M}-Z_{0}^{\prime}\right) S<Z_{0}-H_{0} \\
H_{0}+\mathcal{M} S<Z_{0}+Z_{0}^{\prime} S
\end{gathered}
$$

and the particle is outside the scraper.

Similarly, for $S_{e}<S<L$ we have

$$
\begin{gathered}
\left(\mathcal{M}-Z_{0}^{\prime}\right) S_{e}<\left(\mathcal{M}-Z_{0}^{\prime}\right) S \\
Z_{0}-H_{0}<\left(\mathcal{M}-Z_{0}^{\prime}\right) S \\
Z_{0}+Z_{0}^{\prime} S<H_{0}+\mathcal{M} S
\end{gathered}
$$

and the particle is inside the scraper. The length of the track in the scraper is then

$$
L_{T}=\left(L-S_{e}\right) \sqrt{1+Z_{0}^{\prime 2}}
$$

and the depth is

$$
D_{T}=\left(Z_{L}-H_{L}\right) / 2 .
$$

4. For $Z_{0} \geq H_{0}$ and $Z_{0}^{\prime}>\mathcal{M}$ we have

$$
Z_{0}-H_{0} \geq 0, \quad \mathcal{M}-Z_{0}^{\prime}<0, \quad S_{e} \leq 0 .
$$

It follows that for $0<S$ we have

$$
Z_{0}+Z_{0}^{\prime} S>H_{0}+\mathcal{M} S
$$

and the particle is outside the scraper. 


\section{Energy Loss in the Scraper}

The rates of energy loss (also called stopping power) for charged particles passing through various materials are given in [3]. Here the total stopping power is defined to be the sum of the "collision stopping power" and the "nuclear stopping power". The collision stopping power is the "average rate of energy loss per unit path length, due to coulomb collisions that result in the ionization and excitation of atoms". The nuclear stopping power is the "average rate of energy loss per unit path length due to the transfer of energy to recoiling atoms in elastic collisions". Particles lose energy according to these stopping powers until they either come to rest in the material or undergo an inelastic nuclear interaction. The path length required for the particle to come to rest (with no inelastic nuclear interactions) is called the range.

The rate of energy loss for particles that travel through the scraper material (copper) is

$$
-\frac{d E}{d x}=-q^{2} \frac{d E_{p}}{d x}
$$

where $q$ is the particle charge (in units of proton charge) and $-d E_{p} / d x$ is the rate of energy loss of a proton traveling through the material with the same velocity as the particle. The parameter $x$ is the distance traveled times the density of the material.

For polarized protons at extraction in AGS, the kinetic energy is

$$
W_{p}=22.874 \mathrm{GeV}
$$

and the rate of energy loss in copper is approximately [3]

$$
-\frac{d E_{p}}{d x}=1.6 \mathrm{MeV} \mathrm{cm}^{2} / \mathrm{g} \text {. }
$$

Multiplying by the density of copper,

$$
\rho=8.96 \mathrm{~g} / \mathrm{cm}^{3},
$$

gives the rate of energy loss in units of $\mathrm{MeV} / \mathrm{cm}$. Thus

$$
-\rho \frac{d E_{p}}{d x}=14.3 \mathrm{MeV} / \mathrm{cm}
$$

and the change in energy along a track of length $L_{T}$ is approximately

$$
\Delta E=L_{T}\left\{\rho \frac{d E_{p}}{d x}\right\} .
$$


Taking $L_{T}$ equal to the length $L$ of the scraper gives

$$
\Delta E=-2.9 \mathrm{GeV} \text {. }
$$

The range of the proton in copper is approximately

$$
\mathcal{R}_{p}=W_{p}\left\{-\rho \frac{d E_{p}}{d x}\right\}^{-1}=\underline{15.96 \mathrm{~m}}
$$

which is nearly 8 times the length of the scraper.

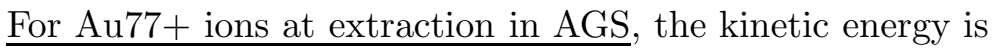

$$
W=1745.42 \mathrm{GeV}
$$

which is $8.86 \mathrm{GeV}$ per nucleon. The kinetic energy of a proton moving with the same velocity as the ion is $8.93 \mathrm{GeV}$. The rate of energy loss of the proton is then [3]

$$
-\frac{d E_{p}}{d x}=1.534 \mathrm{MeV} \mathrm{cm}^{2} / \mathrm{g}
$$

and, with $q=79$, equation (71) gives

$$
-\frac{d E}{d x}=9573.7 \mathrm{MeV} \mathrm{cm}^{2} / \mathrm{g} \text {. }
$$

Multiplying by the density of copper gives a loss rate of

$$
-\rho \frac{d E}{d x}=85.8 \mathrm{GeV} / \mathrm{cm} .
$$

The range of the Au79+ ion in copper is then approximately

$$
\mathcal{R}=W\left\{-\rho \frac{d E}{d x}\right\}^{-1}=\underline{20.3 \mathrm{~cm}} .
$$

This is some two orders of magnitude less than the range (78) of polarized protons at AGS extraction.

\section{Turn-by-Turn Tracking}

Particles that miss the scraper or those that pass through it and continue around the machine are tracked turn-by-turn. We assume as before that the angle $Z^{\prime}$ is unchanged by passage through the scraper. After one turn, 
a particle launched with position $Z_{0}$ and angle $Z_{0}^{\prime}$ at the upstream end of the scraper will have position and angle given by [4]

$$
\mathbf{Z}=\mathbf{M} \mathbf{Z}_{0}+\frac{\Delta p}{p}\left(\mathbf{D}_{0}-\mathbf{M D}_{0}\right)+\left(1-\frac{\Delta p}{p}\right)\left(\mathbf{d}_{0}-\mathbf{M d}_{0}\right)
$$

or equivalently

$$
\mathbf{Z}-\frac{\Delta p}{p} \mathbf{D}_{0}-\left(1-\frac{\Delta p}{p}\right) \mathbf{d}_{0}=\mathbf{M}\left\{\mathbf{Z}_{0}-\frac{\Delta p}{p} \mathbf{D}_{0}-\left(1-\frac{\Delta p}{p}\right) \mathbf{d}_{0}\right\}
$$

where

$$
\begin{array}{cc}
\mathbf{Z}=\left(\begin{array}{c}
Z \\
Z^{\prime}
\end{array}\right), \quad \mathbf{Z}_{0}=\left(\begin{array}{c}
Z_{0} \\
Z_{0}^{\prime}
\end{array}\right) \\
\mathbf{D}_{0}=\left(\begin{array}{c}
D_{0} \\
D_{0}^{\prime}
\end{array}\right), \quad \mathbf{d}_{0}=\left(\begin{array}{c}
d_{0} \\
d_{0}^{\prime}
\end{array}\right)
\end{array}
$$

and

$$
\mathbf{M}=\left(\begin{array}{ll}
M_{11} & M_{12} \\
M_{21} & M_{22}
\end{array}\right) .
$$

Here $D_{0}$ and $D_{0}^{\prime}$ are the periodic dispersion functions at the upstream end of the scraper. The MAD model of the AGS gives

$$
\underline{D_{0}=2.3427} \text { meters, } \quad \underline{D_{0}^{\prime}=-0.1588} .
$$

The elements of the one-turn matrix $\mathbf{M}$ are

$$
\begin{gathered}
M_{11}=\cos \mu+\alpha_{0} \sin \mu, \quad M_{12}=\beta_{0} \sin \mu \\
M_{21}=-\gamma_{0} \sin \mu, \quad M_{22}=\cos \mu-\alpha_{0} \sin \mu
\end{gathered}
$$

where

$$
\mu=2 \pi Q
$$

and $Q$ is the horizontal tune of the machine.

The parameter $\Delta p / p$ is taken to be

$$
\frac{\Delta p}{p}=\frac{1}{\beta_{R}^{2}} \frac{\Delta E}{E}-\frac{\Delta q}{q}
$$

where $\beta_{R}$ is the particle speed divided by the speed of light and $\Delta E / E$ and $\Delta q / q$ are respectively the fractional changes in the particle's energy 
and charge upon passing through the scraper. We assume that any particle emerging from the scraper will have $\Delta q / q \geq 0$. Thus we always have

$$
\frac{\Delta p}{p} \leq 0
$$

If the particle emerges from the scraper with $\Delta p / p$ less negative than a certain amount, the particle will survive its trip around the machine and return to the scraper.

\section{Maximum $\Delta p / p$ for Turn-by-Turn Survival}

As shown in the Appendix, an estimate of the maximum (most negative) $\Delta p / p$ for which the particle always survives and returns to the scraper is

$$
\left(\frac{\Delta p}{p}\right)_{M}=\frac{H_{M}}{D_{M}+\sqrt{\mathcal{E} \beta_{M}}}
$$

where

$$
\mathcal{E} \beta_{0}=\left(D_{0}-d_{0}\right)^{2}+\left\{\alpha_{0}\left(D_{0}-d_{0}\right)+\beta_{0}\left(D_{0}^{\prime}-d_{0}^{\prime}\right)\right\}^{2} .
$$

Here $H_{M}<0$ is a radially inward limiting aperture downstream of the scraper and $\beta_{M}$ and $D_{M}$ are the corresponding beta function and periodic dispersion. Substituting the values of the lattice parameters at the upstream end of the scraper into (96) gives

$$
\sqrt{\mathcal{E} \beta_{0}}=2.5384 \mathrm{~m} \text {. }
$$

This is used to calculate

$$
\sqrt{\mathcal{E} \beta_{M}}=\sqrt{\beta_{M} / \beta_{0}} \sqrt{\mathcal{E} \beta_{0}}
$$

for use in (95).

Substituting $(\Delta p / p)_{M}$ for $\Delta p / p$ in (93) gives the maximum (most negative) fractional energy change

$$
\left(\frac{\Delta E}{E}\right)_{M}=\beta_{R}^{2}\left\{\left(\frac{\Delta p}{p}\right)_{M}+\frac{\Delta q}{q}\right\} .
$$

The path length (in the scraper material) required to achieve this change is approximately

$$
L_{M}=E\left\{\rho \frac{d E}{d x}\right\}^{-1}\left(\frac{\Delta E}{E}\right)_{M} .
$$


We assume that particles emerging from the scraper having traveled along a path length greater than $L_{M}$ will have $\Delta p / p$ too large for a subsequent return to the scraper.

Limiting apertures may occur where the lattice parameter $\beta$ and the periodic dispersion $D$ both reach maximums. At these locations one has

$$
H_{M}=-76.2 \mathrm{~mm}, \quad D_{M}=3 \mathrm{~m}, \quad \beta_{M}=22 \mathrm{~m}
$$

which gives

$$
\sqrt{\mathcal{E} \beta_{M}}=2.8607 \mathrm{~m}
$$

and

$$
\frac{H_{M}}{D_{M}+\sqrt{\mathcal{E} \beta_{M}}}=-0.0130
$$

A limiting aperture may also occur at the injection kicker in the A5 straight section. Here

$$
H_{M}=-64.3 \mathrm{~mm}, \quad D_{M}=2.70 \mathrm{~m}, \quad \beta_{M}=23.2 \mathrm{~m}
$$

and putting in the numbers one obtains

$$
\sqrt{\mathcal{E} \beta_{M}}=2.9376 \mathrm{~m}
$$

and

$$
\frac{H_{M}}{D_{M}+\sqrt{\mathcal{E} \beta_{M}}}=-0.0114
$$

Thus we take

$$
\left(\frac{\Delta p}{p}\right)_{M}=-0.0114
$$

For protons with kinetic energy $W_{p}=22.874 \mathrm{GeV}$ this gives, by substitution into (99) and (100),

$$
\underline{L_{M}=18.9 \mathrm{~cm}} .
$$

Protons that emerge from the scraper having traveled along a path length greater than $L_{M}$ will have $\Delta p / p$ too large for a subsequent return to the scraper. These particles will be lost on an aperture downstream of the scraper. Protons that emerge having traveled along a path length less than $L_{M}$ eventually will hit the scraper again and could travel at most an additional distance $L$ in the scraper before emerging again and finally being 
lost on an aperture downstream. Since the range (78) is larger than $L_{M}+L$, it follows that protons will not stop in the scraper unless there is some mechanism other than stopping power to stop them. In Sections 11 and 12 it is shown that inelastic interactions of protons with copper nuclei in the scraper provide the necessary mechanism.

For Au77+ ions that lose 2 electrons in the scraper we have

$$
\frac{\Delta q}{q}=\frac{79-77}{77}=0.025974
$$

and it follows from (93) and (107) that for any $\Delta E<0$ we have

$$
\frac{\Delta p}{p}<-\frac{2}{77}<\left(\frac{\Delta p}{p}\right)_{M} .
$$

Thus any fully stripped ions (Au79+) that emerge from the scraper will be lost on an aperture downstream of the scraper.

For Au77+ ions that do not lose (or gain) electrons in the scraper we have $\Delta q / q=0$ and equations (99), (100), and (107) give

$$
\underline{L_{M}}=0.25 \mathrm{~cm} .
$$

Because this is so small, most of the Au77+ ions that emerge from the scraper after hitting it once will have $\Delta p / p$ too large for a subsequent return to the scraper. These ions will be lost on an aperture downstream of the scraper. However, in Sections 8 and 9 it will be shown that on the first hit a significant fraction of the Au77+ ions have track lengths greater than the range (83). These ions will be stopped in the scraper.

\section{Particle Tracks on First Hit on Scraper}

As the circulating beam is moved radially inward at the scraper, particles in the distribution of Figure 4 hit the scraper on various turns around the machine. The rate at which the orbit is moved inward at the upstream end of the scraper is taken to be $1 \mathrm{~mm} / \mathrm{ms}$. For protons with a kinetic energy of $22.874 \mathrm{GeV}$ and for Au77+ ions with a kinetic energy of $8.86 \mathrm{GeV}$ per nucleon, this amounts to $0.0027 \mathrm{~mm}$ per turn. Each of the 20055 particles in the distribution will hit the scraper at least once. After a hit, any 
particle that continues around the machine will eventually hit the scraper again if it is not lost on some other aperture. We assume again that the angle $Z^{\prime}$ is unchanged by the passage of a particle through the scraper.

Each time a given particle hits the scraper the track length $L_{T}$ and depth $D_{T}$ (as calculated in Section 4) are recorded along with the number of the turn on which the hit occurs. Figure 5 shows a scatter plot of $L_{T}$ and turn number for each particle's first hit on the scraper. Here the machine tune $Q=8.71$. Figure 6 shows the corresponding plot of $D_{T}$ and turn number. Figures $\mathbf{7}$ and $\mathbf{8}$ are the same plots for the case in which $Q=8.75$. The average $L_{T}$ and $D_{T}$ obtained for these and several other tunes are listed in Table 1 . Here we see that for fractional tunes $2 / 3,3 / 4$ and $4 / 5$, the track lengths and depths are considerably less.

Table 1: First-hit parameters obtained for various tunes.

\begin{tabular}{|c|c|c|c|c|}
\hline$Q$ & $\begin{array}{c}<L_{T}> \\
(\mathrm{mm})\end{array}$ & $\begin{array}{c}<D_{T}> \\
(\mathrm{mm})\end{array}$ & $\begin{array}{c}F_{1}\left(L_{M}\right) \\
(\mathrm{H}+)\end{array}$ & $\begin{array}{c}F_{1}(\mathcal{R}) \\
(\mathrm{Au} 77+)\end{array}$ \\
\hline 8.6667 & 137 & -0.00196 & 0.1475 & 0.1373 \\
\hline 8.71 & 413 & -0.0145 & 0.7125 & 0.6919 \\
8.72 & 518 & -0.0146 & 0.7691 & 0.7527 \\
8.73 & 518 & -0.0148 & 0.7596 & 0.7428 \\
8.74 & 446 & -0.0148 & 0.7298 & 0.7125 \\
\hline 8.75 & 199 & -0.00266 & 0.2272 & 0.2126 \\
\hline 8.76 & 511 & -0.0139 & 0.7463 & 0.7271 \\
8.77 & 494 & -0.0120 & 0.7144 & 0.6973 \\
8.78 & 589 & -0.0147 & 0.7700 & 0.7545 \\
8.79 & 425 & -0.0126 & 0.6996 & 0.6785 \\
\hline 8.80 & 272 & -0.00337 & 0.3236 & 0.3035 \\
\hline
\end{tabular}

We define the function $F_{1}(X)$ to be the fraction of first-hit particles for which track length

$$
L_{T} \geq X
$$

Taking $X=L_{M}$, where $L_{M}$ is given by (108), gives the fraction $F_{1}\left(L_{M}\right)$ of first-hit protons with track lengths for which $\Delta p / p$ is too large to allow a second hit on the scraper. If these particles emerge from the scraper after the first hit, they will be lost on an aperture downstream of the scraper. Table 1 lists the values of $F_{1}\left(L_{M}\right)$ for various tunes. We again see that the numbers are considerably less for fractional tunes $2 / 3,3 / 4$ and $4 / 5$. 
Taking $X=\mathcal{R}$, where $\mathcal{R}$ is given by (83), gives the fraction $F_{1}(\mathcal{R})$ of first-hit Au77+ ions with track lengths greater than the range of the ions in copper. These ions are stopped in the scraper. Table 1 lists the values of $F_{1}(\mathcal{R})$ for various tunes. We again see that the numbers are considerably less for fractional tunes $2 / 3,3 / 4$ and $4 / 5$.

\section{Fraction of Au77+ Ions Lost in and downstream of the Scraper}

The values of $F_{1}(\mathcal{R})$ listed in Table 1 show that (except for fractional tunes $2 / 3,3 / 4$ and $4 / 5$ ) the fraction of Au77+ ions stopped in the scraper on the first hit ranges from $\underline{0.68 \text { to } 0.75}$. Most of the ions that do not stop will emerge from the scraper with charge $q=79$ and most will have traveled at least $0.25 \mathrm{~cm}$ in the scraper material. According to (110) and (111), they will have $\Delta p / p$ too large to return to the scraper for a second hit. The fraction of first-hit ions lost downstream of the scraper then ranges from $\underline{0.25}$ to 0.32 .

If the rate at which the orbit is moved inward at the upstream end of the scraper is increased from 1 to $2 \mathrm{~mm} / \mathrm{ms}$, then the maximum fraction of

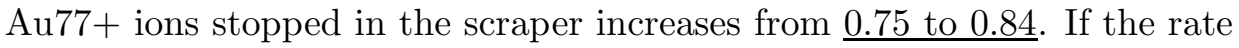
is decreased from 1 to $0.5 \mathrm{~mm} / \mathrm{ms}$, then the maximum fraction stopped decreases from $\underline{0.75 \text { to } 0.63}$.

\section{Proton Tracks on Second Hit on Scraper}

The fraction of first-hit protons that may go on to hit the scraper a second time is $1-F_{1}\left(L_{M}\right)$. These are the particles for which $\Delta p / p$ is small enough to allow a second hit. The track lengths and depths (as calculated in Section 4) for the second-hit particles are recorded as before along with the number of the turn on which the hit occurs. For each particle the sum $\Sigma L_{T}$ of the first and second-hit track lengths is calculated. A scatter plot of $\Sigma L_{T}$ and turn number is shown in Figure 9 for the case in which machine tune $Q=8.71$. The corresponding plot of $D_{T}$ and turn number is shown in Figure 10.

Figures 11 and 12 are the same plots for the case in which $Q=8.75$. The average $\Sigma L_{T}$ and $D_{T}$ obtained for these and several other tunes are listed in Table 2. Here we again see that for fractional tunes $2 / 3,3 / 4$ and $4 / 5$, 
the track lengths and depths are less.

Comparing the Table $\mathbf{2}$ numbers with those in Table $\mathbf{1}$, we see that the second-hit track depths are much larger than the first-hit depths. This is due to the $\Delta p / p$ acquired on the first hit and the nonzero dispersion in the scraper straight.

Table 2: Second-hit parameters obtained for various tunes.

\begin{tabular}{|c|c|c|c|}
\hline$Q$ & $\begin{array}{c}<\Sigma L_{T}> \\
(\mathrm{mm})\end{array}$ & $\begin{array}{c}<D_{T}> \\
(\mathrm{mm})\end{array}$ & $\begin{array}{c}F_{2}\left(L_{M}\right) \\
(\mathrm{H}+)\end{array}$ \\
\hline 8.6667 & 1829 & -8.97 & 0.8921 \\
\hline 8.71 & 2054 & -16.1 & 0.9969 \\
8.72 & 2054 & -15.2 & 0.9963 \\
8.73 & 2042 & -14.1 & 0.9934 \\
8.74 & 2053 & -14.1 & 0.9967 \\
\hline 8.75 & 1938 & -9.02 & 0.9419 \\
\hline 8.76 & 2056 & -13.0 & 0.9957 \\
8.77 & 2045 & -11.9 & 0.9888 \\
8.78 & 2043 & -11.0 & 0.9918 \\
8.79 & 2059 & -10.9 & 0.9973 \\
\hline 8.80 & 1982 & -8.04 & 0.9637 \\
\hline
\end{tabular}

We define the function $F_{2}(X)$ to be the fraction of second-hit particles for which

$$
\Sigma L_{T} \geq X
$$

Taking $X=L_{M}$, where $L_{M}$ is given by (108), gives the fraction of second-hit protons with track lengths for which $\Delta p / p$ is too large to allow a third hit on the scraper. If these particles emerge from the scraper after the second hit, they will be lost on an aperture downstream of the scraper. Table 2 lists the values of $F_{2}\left(L_{M}\right)$ for various tunes. These values are all very close to 1 except for fractional tunes $2 / 3,3 / 4$ and $4 / 5$. 


\section{Inelastic Nuclear Interactions}

For high-energy protons in a given material, the mean free path for inelastic nuclear interactions is given by the empirical formula [5]

$$
\lambda=\frac{1}{\rho} 40.0 A^{0.3}
$$

where $A$ is the atomic weight of the material and $\rho$ is the density in units of $\mathrm{g} / \mathrm{cm}^{3}$. The units of $\lambda$ are $\mathrm{cm}$. For copper we have $A=63$ and

$$
\lambda=15.47 \mathrm{~cm} .
$$

The fraction of protons that have an inelastic nuclear interaction upon traveling a distance $X$ in the material is

$$
f(X)=1-\exp (-X / \lambda)
$$

Taking $X=L_{M}$, where $L_{M}$ is given by (108), gives fraction

$$
\underline{f\left(L_{M}\right)=0.7052 .}
$$

\section{Fraction of Protons Lost in and downstream of the Scraper}

The fraction of first-hit protons that undergo an inelastic nuclear interaction in the scraper is then at least

$$
\mathcal{F}_{1}=f\left(L_{M}\right) F_{1}\left(L_{M}\right)
$$

where $F_{1}(X)$ is defined in Section 8 . We consider these particles to be lost in the scraper. The fraction of first-hit protons that are lost downstream of the scraper is then at most

$$
\mathcal{L}_{1}=\left\{1-f\left(L_{M}\right)\right\} F_{1}\left(L_{M}\right) .
$$

Similarly, the fraction of second-hit protons that undergo an inelastic nuclear interaction in the scraper is at least

$$
\mathcal{F}_{2}=f\left(L_{M}\right) F_{2}\left(L_{M}\right)
$$


and the fraction that are lost downstream of the scraper is at most

$$
\mathcal{L}_{2}=\left\{1-f\left(L_{M}\right)\right\} F_{2}\left(L_{M}\right)
$$

where $F_{2}(X)$ is defined in Section 10 .

Continuing in this way, the fraction of nth-hit protons that undergo an inelastic nuclear interaction in the scraper is at least

$$
\mathcal{F}_{n}=f\left(L_{M}\right) F_{n}\left(L_{M}\right)
$$

and the fraction that are lost downstream of the scraper is at most

$$
\mathcal{L}_{n}=\left\{1-f\left(L_{M}\right)\right\} F_{n}\left(L_{M}\right)
$$

The total fraction of first, second, and third-hit protons that undergo an inelastic nuclear interaction is then at least

$$
\mathcal{F}=\mathcal{F}_{1}+\left(1-F_{1}\right) \mathcal{F}_{2}+\left(1-F_{1}\right)\left(1-F_{2}\right) \mathcal{F}_{3}
$$

and the total fraction of protons lost downstream of the scraper is at most

$$
\mathcal{L}=\mathcal{L}_{1}+\left(1-F_{1}\right) \mathcal{L}_{2}+\left(1-F_{1}\right)\left(1-F_{2}\right) \mathcal{L}_{3} .
$$

Using (118-123) we have

$$
\mathcal{F}=f\left\{F_{1}+\left(1-F_{1}\right) F_{2}+\left(1-F_{1}\right)\left(1-F_{2}\right) F_{3}\right\}
$$

and

$$
\mathcal{L}=(1-f)\left\{F_{1}+\left(1-F_{1}\right) F_{2}+\left(1-F_{1}\right)\left(1-F_{2}\right) F_{3}\right\}
$$

Except for fractional tunes $2 / 3,3 / 4$, and $4 / 5$ we see from the numbers listed in Table 2 that $F_{2}\left(L_{M}\right)$ is very close to 1 . Thus we have simply

$$
\mathcal{F}=f\left(L_{M}\right)
$$

and

$$
\mathcal{L}=1-f\left(L_{M}\right)
$$

where $f\left(L_{M}\right)$ is given by (117). The fraction of protons that undergo an inelastic nuclear interaction is then at least

$$
\underline{\mathcal{F}=0.7052}
$$


and the fraction that are lost downstream of the scraper is at most

$$
\underline{\mathcal{L}}=0.2948 .
$$

These lower and upper bounds are independent of the rate at which the circulating beam is moved into the scraper. However, if there were a fast dipole magnet to kick the circulating beam into the dump all at once then the fraction of protons that would undergo an inelastic nuclear interaction would be given by (116) with $X=L$. This gives a fraction very close to $100 \%$.

\section{Multiple Coulomb Scattering}

So far we have assumed that the angle $Z^{\prime}$ does not change as a particle passes through the scraper material. Here we consider a refinement in which a simple random walk simulates changes in the angle due to multiple coulomb scattering. In this case a series of steps

$$
S_{i+1}=S_{i}+\Delta S
$$

in the coordinate $S$ are taken with the particle position and angle at $S_{i+1}$ given by

$$
Z_{i+1}=Z_{i}+Z_{i}^{\prime} \Delta S
$$

and

$$
Z_{i+1}^{\prime}=Z_{i}^{\prime} \pm \phi
$$

Here $\Delta S$ is the step size, $Z_{i}$ and $Z_{i}^{\prime}$ are the position and angle at $S_{i}$, and $\phi$ is a fixed angular kick. For each step the sign of $\phi$ is chosen randomly with $50 \%$ probability of getting a given sign. Steps are taken until the particle exits the scraper. Let $S_{X}$ be the $S$ coordinate at the exit point and let $Z_{X}$ and $Z_{X}^{\prime}$ be the position and angle of the particle there. Then, in the turn-by-turn equations of Section $6, Z_{0}$ and $Z_{0}^{\prime}$ must be replaced with $Z_{I}$ and $Z_{X}^{\prime}$, respectively, where

$$
Z_{I}=Z_{X}-S_{X} Z_{X}^{\prime}
$$

Taking

$$
\Delta S=1.0 \mathrm{~mm}
$$

and

$$
\phi=25 \text { microradians }
$$


Table 3: First-hit parameters obtained with scattering simulation.

\begin{tabular}{|c|c|c|c|c|}
\hline$Q$ & $\begin{array}{c}<L_{T}> \\
(\mathrm{mm})\end{array}$ & $\begin{array}{c}<D_{T}> \\
(\mathrm{mm})\end{array}$ & $\begin{array}{c}F_{1}\left(L_{M}\right) \\
(\mathrm{H}+)\end{array}$ & $\begin{array}{c}F_{1}(\mathcal{R}) \\
(\mathrm{Au} 77+)\end{array}$ \\
\hline 8.6667 & 253 & -0.0318 & 0.1962 & 0.1865 \\
\hline 8.71 & 753 & -0.1187 & 0.6347 & 0.6101 \\
8.72 & 781 & -0.1234 & 0.6643 & 0.6415 \\
8.73 & 795 & -0.1249 & 0.6640 & 0.6400 \\
8.74 & 759 & -0.1204 & 0.6436 & 0.6252 \\
\hline 8.75 & 347 & -0.0455 & 0.2820 & 0.2673 \\
\hline 8.76 & 774 & -0.1219 & 0.6480 & 0.6277 \\
8.77 & 758 & -0.1195 & 0.6221 & 0.5960 \\
8.78 & 815 & -0.1274 & 0.6741 & 0.6501 \\
8.79 & 746 & -0.1171 & 0.6139 & 0.5915 \\
\hline 8.80 & 447 & -0.0607 & 0.3680 & 0.3514 \\
\hline
\end{tabular}

we obtain the numbers listed in Table 3 for various tunes. Comparing with the numbers listed in Table 1 we see that the fraction $F_{1}(\mathcal{R})$ of $\mathrm{Au} 77+$ ions that stop in the scraper is reduced from a maximum of 0.75 to 0.65. This gives an indication of the effect of multiple coulomb scattering.

\section{Appendix}

Let

$$
\begin{array}{cc}
\mathbf{Z}=\left(\begin{array}{c}
Z \\
Z^{\prime}
\end{array}\right), & \mathbf{Z}_{0}=\left(\begin{array}{c}
Z_{0} \\
Z_{0}^{\prime}
\end{array}\right) \\
\mathbf{D}=\left(\begin{array}{c}
D \\
D^{\prime}
\end{array}\right), & \mathbf{D}_{0}=\left(\begin{array}{c}
D_{0} \\
D_{0}^{\prime}
\end{array}\right) \\
\mathbf{d}=\left(\begin{array}{c}
d \\
d^{\prime}
\end{array}\right), & \mathbf{d}_{0}=\left(\begin{array}{c}
d_{0} \\
d_{0}^{\prime}
\end{array}\right)
\end{array}
$$

and

$$
\mathbf{E}=\left(\begin{array}{rr}
\beta & -\alpha \\
-\alpha & \gamma
\end{array}\right), \quad \mathbf{E}_{0}=\left(\begin{array}{rr}
\beta_{0} & -\alpha_{0} \\
-\alpha_{0} & \gamma_{0}
\end{array}\right)
$$


where the parameters with subscript 0 are as defined previously and the parameters with no subscript are the corresponding parameters at some location downstream of the scraper.

Then

$$
\mathbf{E}=\mathbf{N} \mathbf{E}_{0} \mathbf{N}^{\dagger}
$$

and [4]

$$
\mathbf{Z}=\mathbf{N} Z_{0}+\frac{\Delta p}{p}\left(\mathbf{D}-\mathbf{N D}_{0}\right)+\left(1-\frac{\Delta p}{p}\right)\left(\mathbf{d}-\mathbf{N d}_{0}\right)
$$

or equivalently

$$
\mathbf{Z}-\frac{\Delta p}{p} \mathbf{D}-\left(1-\frac{\Delta p}{p}\right) \mathbf{d}=\mathbf{N}\left\{\mathbf{Z}_{0}-\frac{\Delta p}{p} \mathbf{D}_{0}-\left(1-\frac{\Delta p}{p}\right) \mathbf{d}_{0}\right\}
$$

where $\mathbf{N}$ is the transfer matrix from the upstream end of the scraper to the downstream location. (Here a dagger denotes the transpose of a vector or matrix.)

Defining

$$
\mathbf{X}=\mathbf{Z}-\mathbf{d}-\frac{\Delta p}{p}(\mathbf{D}-\mathbf{d})
$$

and

$$
\mathbf{X}_{0}=\mathbf{Z}_{0}-\mathbf{d}_{0}-\frac{\Delta p}{p}\left(\mathbf{D}_{0}-\mathbf{d}_{0}\right)
$$

we have

$$
\mathbf{X}=\mathbf{N X}_{0}
$$

and therefore

$$
\mathbf{X}^{\dagger} \mathbf{E}^{-1} \mathbf{X}=\mathbf{X}_{0}^{\dagger} \mathbf{N}^{\dagger} \mathbf{E}^{-1} \mathbf{N} \mathbf{X}_{0}
$$

Taking the inverse of (142) gives

$$
\mathbf{E}^{-1}=\left(\mathbf{N}^{\dagger}\right)^{-1} \mathbf{E}_{0}^{-1} \mathbf{N}^{-1}
$$

and (148) becomes

where

$$
\mathbf{X}^{\dagger} \mathbf{E}^{-1} \mathbf{X}=\mathbf{X}_{0}^{\dagger} \mathbf{E}_{0}^{-1} \mathbf{X}_{0}
$$

$$
\begin{aligned}
\mathbf{X}_{0}^{\dagger} \mathbf{E}_{0}^{-1} \mathbf{X}_{0} & =\left(\mathbf{Z}_{0}-\mathbf{d}_{0}\right)^{\dagger} \mathbf{E}_{0}^{-1}\left(\mathbf{Z}_{0}-\mathbf{d}_{0}\right) \\
& -\frac{\Delta p}{p}\left(\mathbf{Z}_{0}-\mathbf{d}_{0}\right)^{\dagger} \mathbf{E}_{0}^{-1}\left(\mathbf{D}_{0}-\mathbf{d}_{0}\right) \\
& -\frac{\Delta p}{p}\left(\mathbf{D}_{0}-\mathbf{d}_{0}\right)^{\dagger} \mathbf{E}_{0}^{-1}\left(\mathbf{Z}_{0}-\mathbf{d}_{0}\right) \\
& +\left(\frac{\Delta p}{p}\right)^{2}\left(\mathbf{D}_{0}-\mathbf{d}_{0}\right)^{\dagger} \mathbf{E}_{0}^{-1}\left(\mathbf{D}_{0}-\mathbf{d}_{0}\right)
\end{aligned}
$$


The right and left hand sides of (150) are ellipses that are matched to the machine lattice at the upstream end of the scraper and at the downstream location respectively.

Let

$$
\mathcal{E}=\mathbf{X}_{0}^{\dagger} \mathbf{E}_{0}^{-1} \mathbf{X}_{0}\left(\frac{\Delta p}{p}\right)^{-2}
$$

Then

$$
\mathbf{X}^{\dagger} \mathbf{E}^{-1} \mathbf{X}=\left(\frac{\Delta p}{p}\right)^{2} \mathcal{E}
$$

and doing the matrix multiplication one finds that

$$
X^{2}+\left(\alpha X+\beta X^{\prime}\right)^{2}=\left(\frac{\Delta p}{p}\right)^{2} \mathcal{E} \beta
$$

where

$$
\begin{gathered}
X=Z-d-\frac{\Delta p}{p}(D-d) \\
X^{\prime}=Z^{\prime}-d^{\prime}-\frac{\Delta p}{p}\left(D^{\prime}-d^{\prime}\right) .
\end{gathered}
$$

It follows that

$$
X^{2} \leq\left(\frac{\Delta p}{p}\right)^{2} \mathcal{E} \beta
$$

and for

$$
\frac{\Delta p}{p}<0
$$

we have

$$
\frac{\Delta p}{p} \sqrt{\mathcal{E} \beta} \leq Z-d-\frac{\Delta p}{p}(D-d) \leq-\frac{\Delta p}{p} \sqrt{\mathcal{E} \beta} .
$$

We assume that at the downstream location, the orbit distortion due to dipole perturbations is zero. Thus

$$
d=0
$$

and

$$
\frac{\Delta p}{p}(D+\sqrt{\mathcal{E} \beta}) \leq Z \leq \frac{\Delta p}{p}(D-\sqrt{\mathcal{E} \beta}) .
$$

Let

$$
H_{I}<0, \quad H_{O}>0
$$


be the radial inside and outside apertures at the downstream location. Then circulating beam particles will not hit the aperture if

$$
H_{I}<Z<H_{O}
$$

If $\Delta p / p$ is such that

$$
H_{I} \leq \frac{\Delta p}{p}(D+\sqrt{\mathcal{E} \beta})
$$

and

$$
\frac{\Delta p}{p}(D-\sqrt{\mathcal{E} \beta}) \leq H_{O}
$$

then (163) will be satisfied. Since

$$
0<D+\sqrt{\mathcal{E} \beta}
$$

we may write (164) as

$$
\frac{H_{I}}{D+\sqrt{\mathcal{E} \beta}} \leq \frac{\Delta p}{p}
$$

Up to this point no approximations have been made. Let us now assume that $\mathbf{Z}_{0}$ is sufficiently close to $\mathbf{d}_{0}$ to allow setting

$$
\mathbf{Z}_{0}=\mathbf{d}_{0}
$$

in (151). We then have

$$
\begin{aligned}
\mathbf{X}_{0}^{\dagger} \mathbf{E}_{0}^{-1} \mathbf{X}_{0} & =\left(\frac{\Delta p}{p}\right)^{2}\left(\mathbf{D}_{0}-\mathbf{d}_{0}\right)^{\dagger} \mathbf{E}_{0}^{-1}\left(\mathbf{D}_{0}-\mathbf{d}_{0}\right) \\
\mathcal{E} & =\left(\mathbf{D}_{0}-\mathbf{d}_{0}\right)^{\dagger} \mathbf{E}_{0}^{-1}\left(\mathbf{D}_{0}-\mathbf{d}_{0}\right)
\end{aligned}
$$

and

$$
\mathcal{E} \beta_{0}=\left(D_{0}-d_{0}\right)^{2}+\left\{\alpha_{0}\left(D_{0}-d_{0}\right)+\beta_{0}\left(D_{0}^{\prime}-d_{0}^{\prime}\right)\right\}^{2} .
$$

Using

$$
\mathcal{E} \beta=\frac{\beta}{\beta_{0}} \mathcal{E} \beta_{0}
$$

in (167) then gives the sought-after estimate of the lower bound on $\Delta p / p$. 


\section{References}

[1] C.J. Gardner, "Notes on Dumping Gold Beam in the AGS", C-A/AP/Note 396, August 2010, p. 4

[2] C.J. Gardner, "Notes on Dumping Gold Beam in the AGS", C-A/AP/Note 396, August 2010, pp. 5-6

[3] M.J. Berger, J.S. Coursey, M.A. Zucker and J. Chang, "Stopping-Power and Range Tables for Electrons, Protons, and Helium Ions", www.nist.gov/physlab/data/star/index.cfm

[4] C.J. Gardner, "Notes on Dumping Gold Beam in the AGS", C-A/AP/Note 396, August 2010, pp. 7-9

[5] A.H. Sullivan, "A Guide to Radiation and Radioactivity Levels near High Energy Particle Accelerators", Nuclear Technology Publishing, pp. 6-7 (1992) 


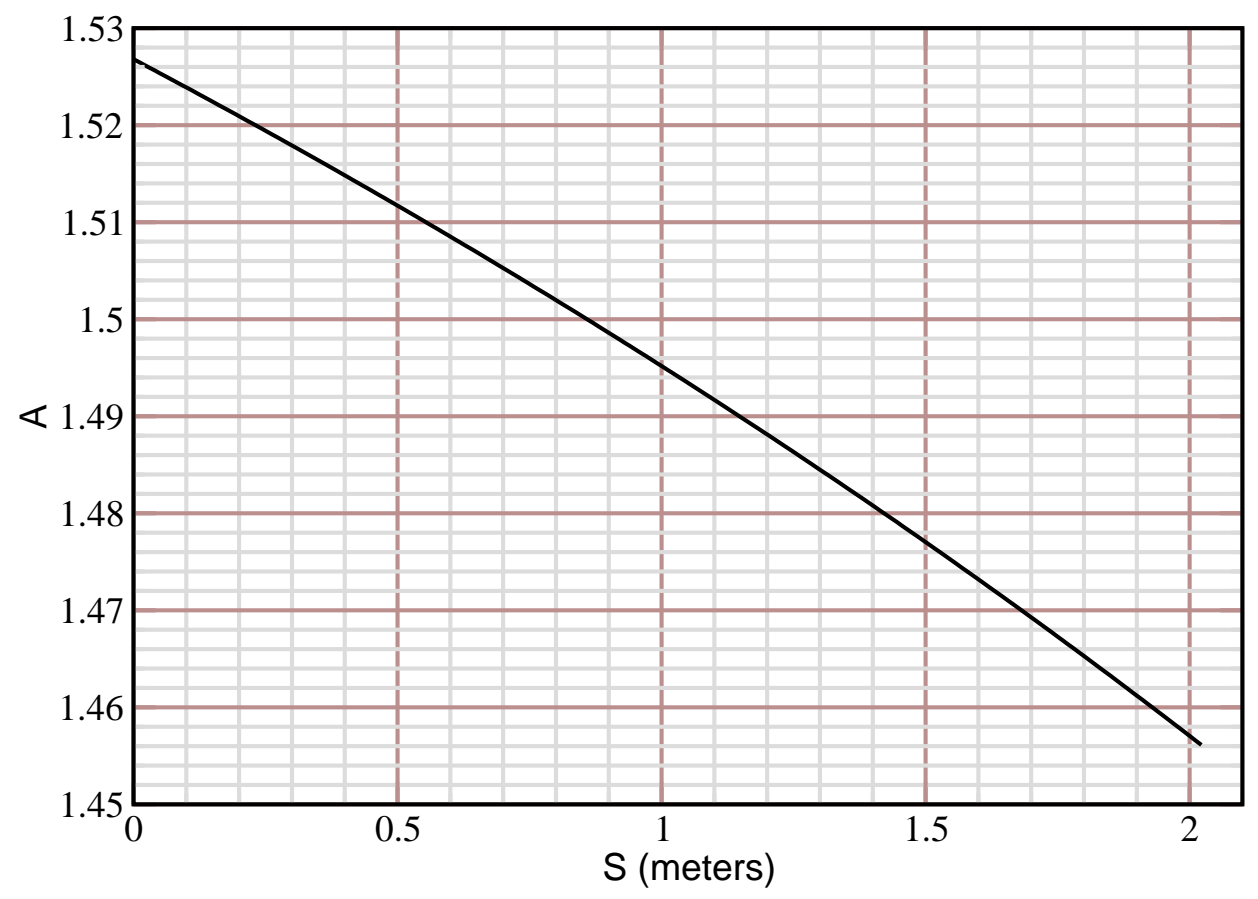

Figure 1: Plot of $\mathcal{A}(S)=\left(\beta_{0}-\sqrt{\beta \beta_{0}}\right) / S$ for $0 \leq S \leq L$. Here $\beta \beta_{0}=$ $\left(\beta_{0}-\alpha_{0} S\right)^{2}+S^{2}$ where $\alpha_{0}, \beta_{0}$ are the lattice parameters at the upstream end of the scraper. The function $\mathcal{A}(S)$ is monotonically decreasing with $\mathcal{A}(0)=\alpha_{0}$ and $\mathcal{A}(L)=1.4561$. Note also that $\mathcal{A}(S)-k_{0}>0$, where $k_{0}$ is given by (22). 


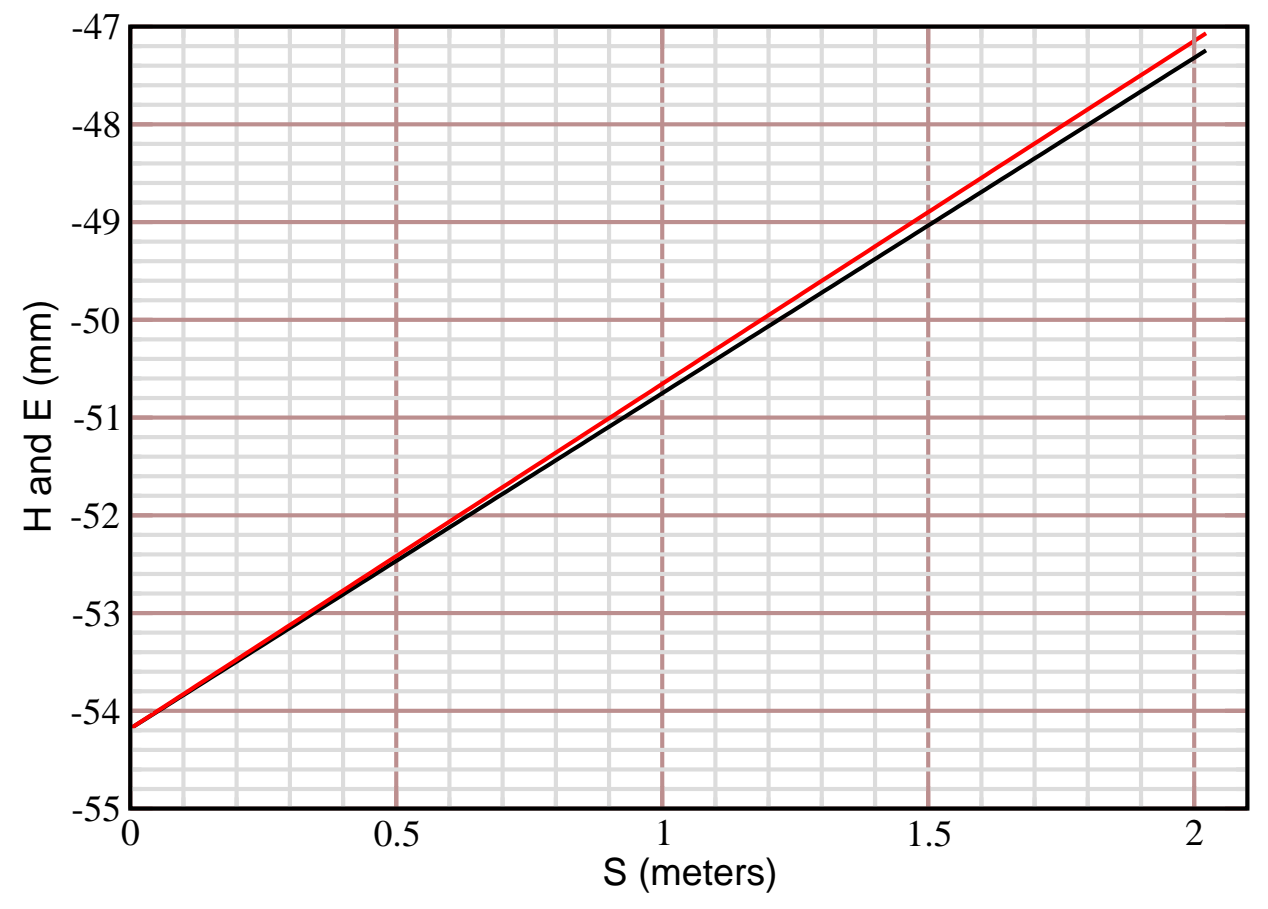

Figure 2: Plot of circulating beam envelope $E(S)$ (upper curve in red) and scraper aperture $H(S)$ (lower curve in black) for $0 \leq S \leq L$. Here $E(S)$ and $H(S)$ are given by (34) and (38), respectively, and we have taken circulating beam emittance $\pi \epsilon=1.0 \pi \mathrm{mm}$ milliradians. The beam envelope touches the scraper aperture at the upstream end and is inside the aperture at all points downstream. 


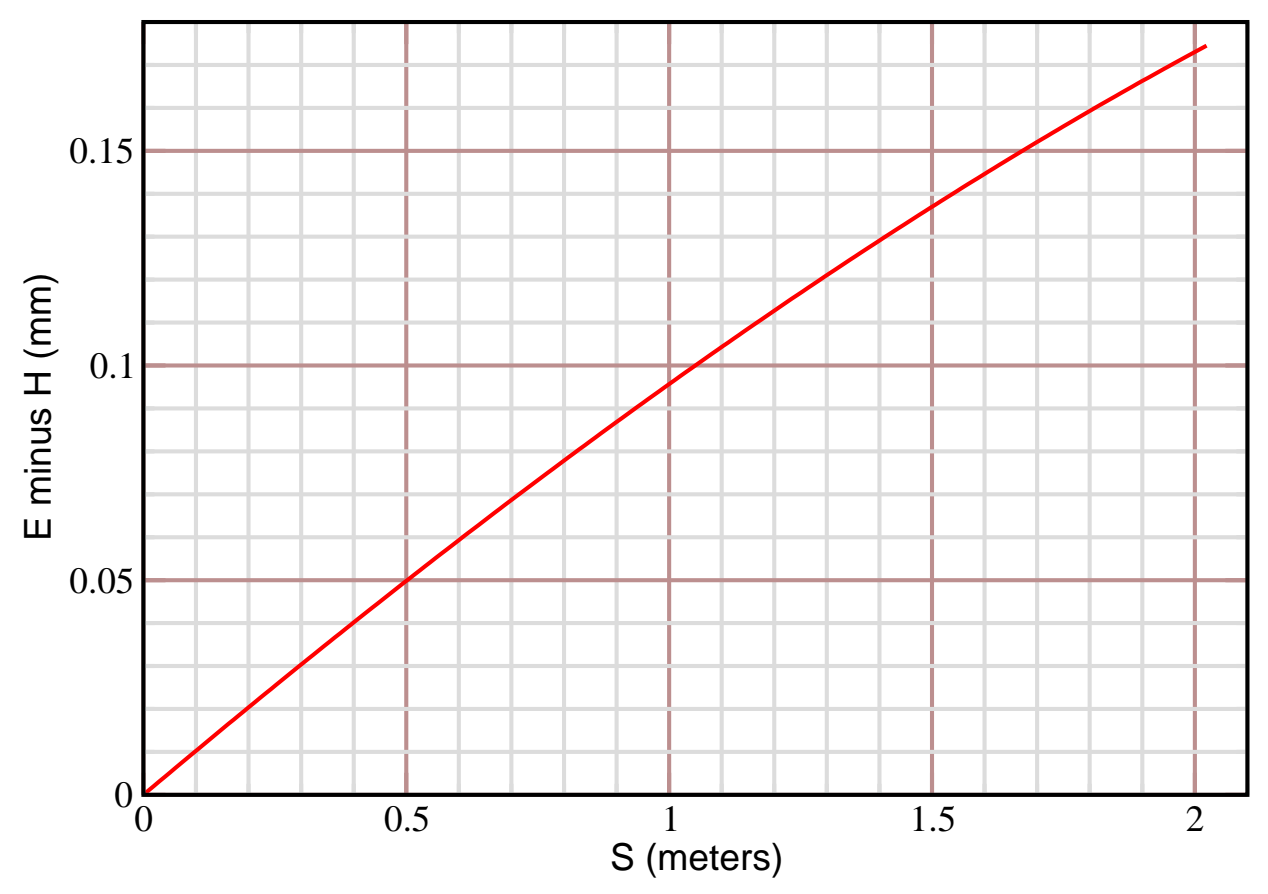

Figure 3: Plot of the separation $E(S)-H(S)$ between envelope and aperture for the case in which $\epsilon=1.0 \mathrm{~mm}$ milliradians. The beam envelope touches the scraper aperture at the upstream end and is inside the aperture at all points downstream. 


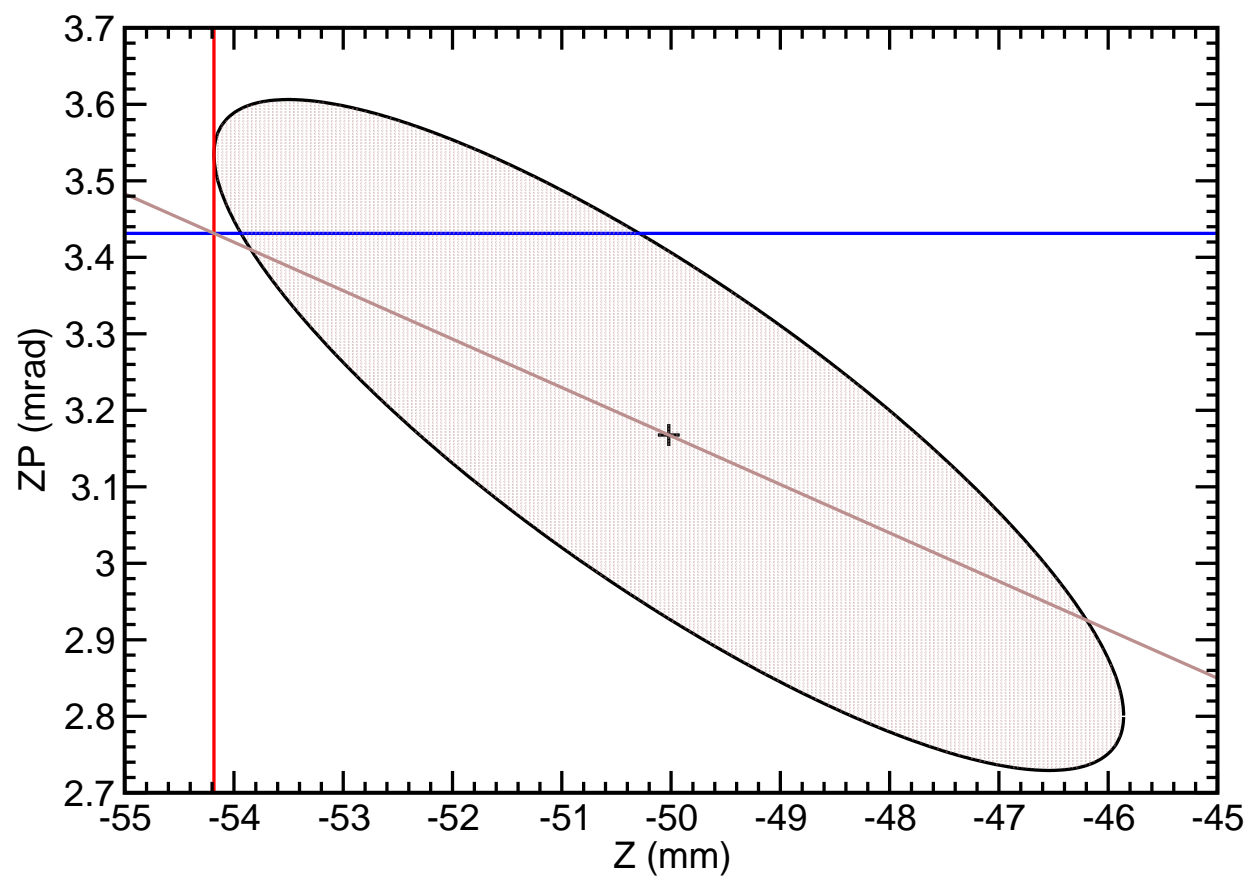

Figure 4: Beam distribution at the upstream end of the scraper at the start of scraping. The border of the distribution is the ellipse (40) which is centered on the equilibrium orbit and matched to the machine lattice. Here we have taken $\epsilon=1.0 \mathrm{~mm}$ milliradians. The ellipse is uniformly covered with 20055 particles. The red line marks the scraper aperture $H_{0}$ and the blue line marks the scraper slope given by (37). The circulating beam moves into the scraper as the ellipse center moves along the brown line given by (36). The red and blue lines divide the plot into quadrants which correspond to the four cases discussed in Section 4. 


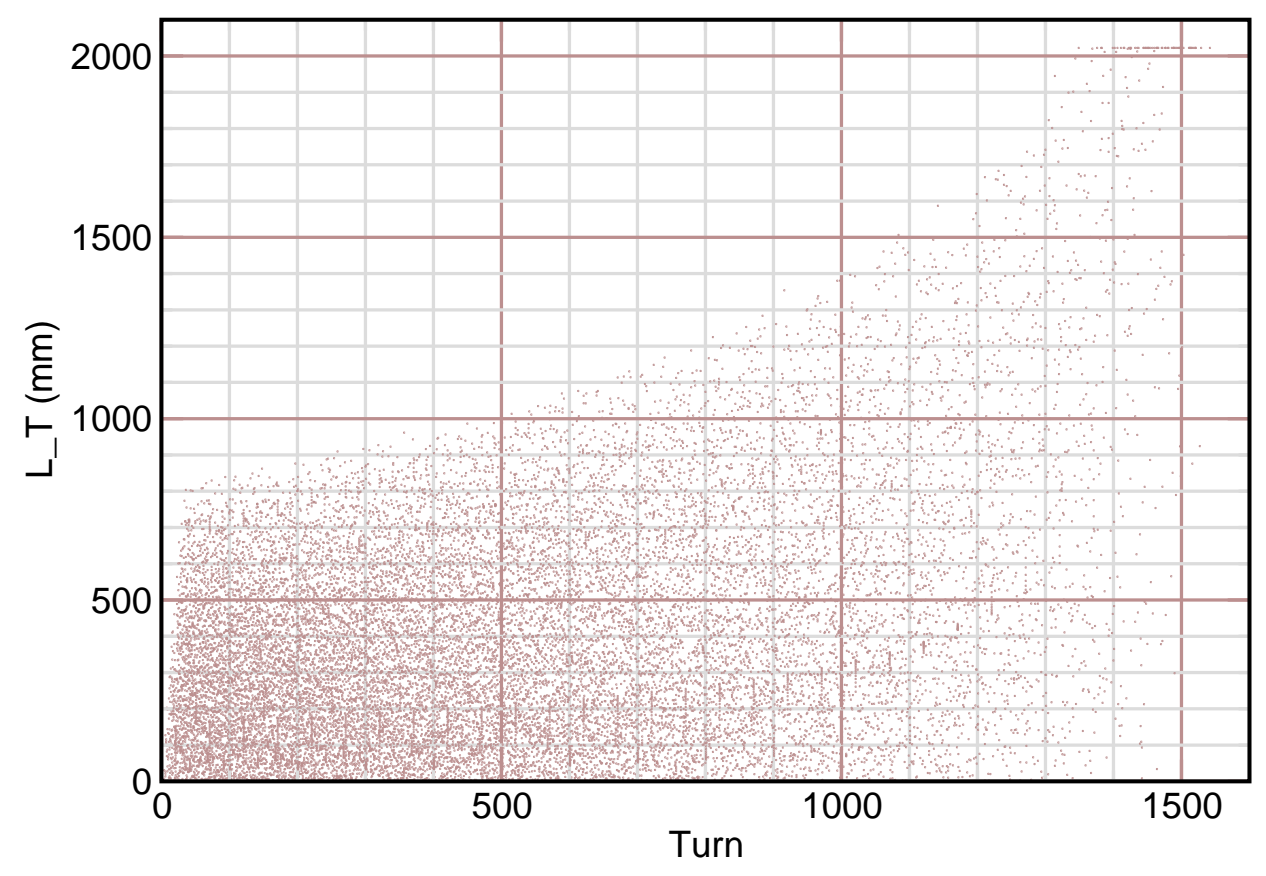

Figure 5: Scatter plot of track length $L_{T}$ and turn number for each particle's first hit on the scraper. Here the machine tune $Q=8.71$. The average $L_{T}$ is $\underline{413 \mathrm{~mm}}$. 


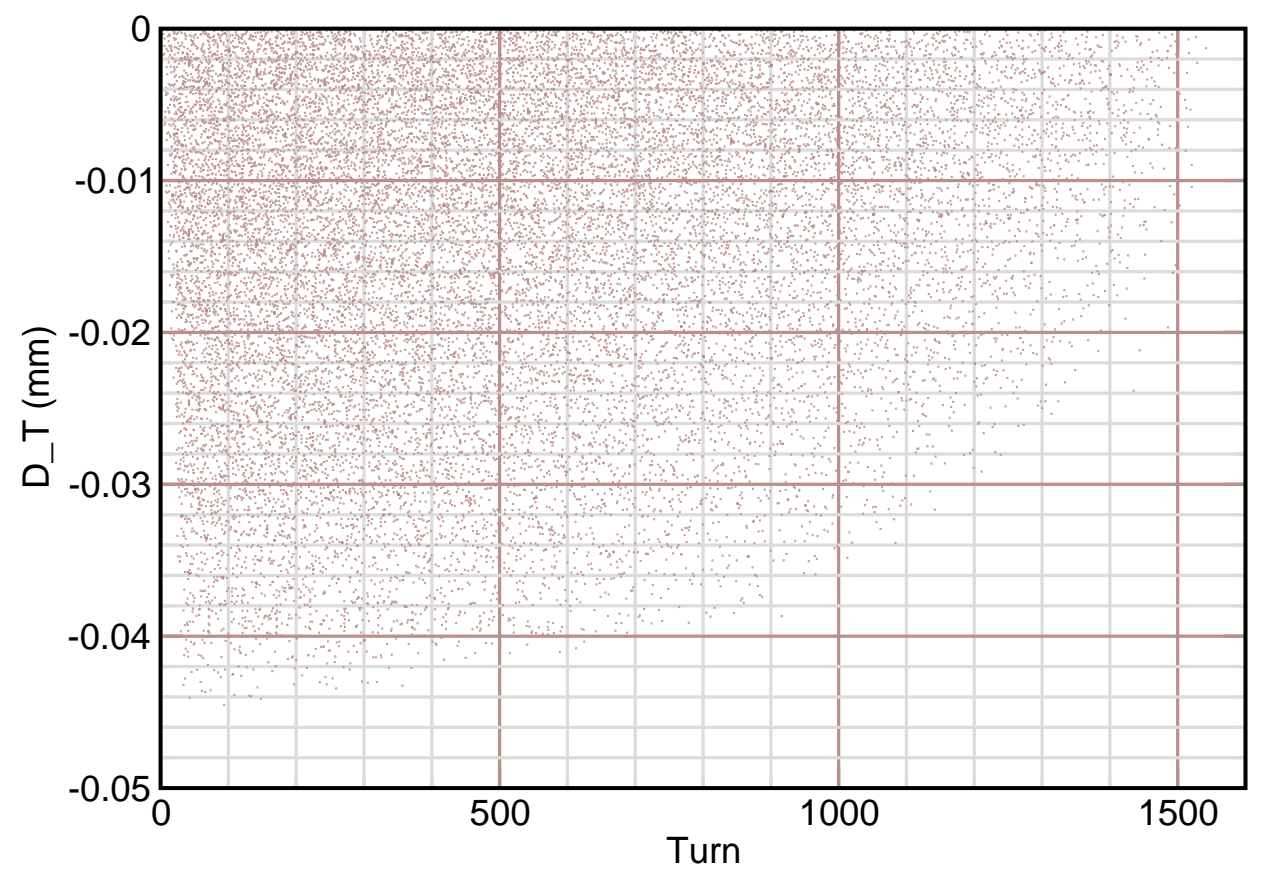

Figure 6: Scatter plot of track depth $D_{T}$ and turn number for each particle's first hit on the scraper. The machine tune $Q=8.71$. The average $D_{T}$ is $-0.0145 \mathrm{~mm}$. 


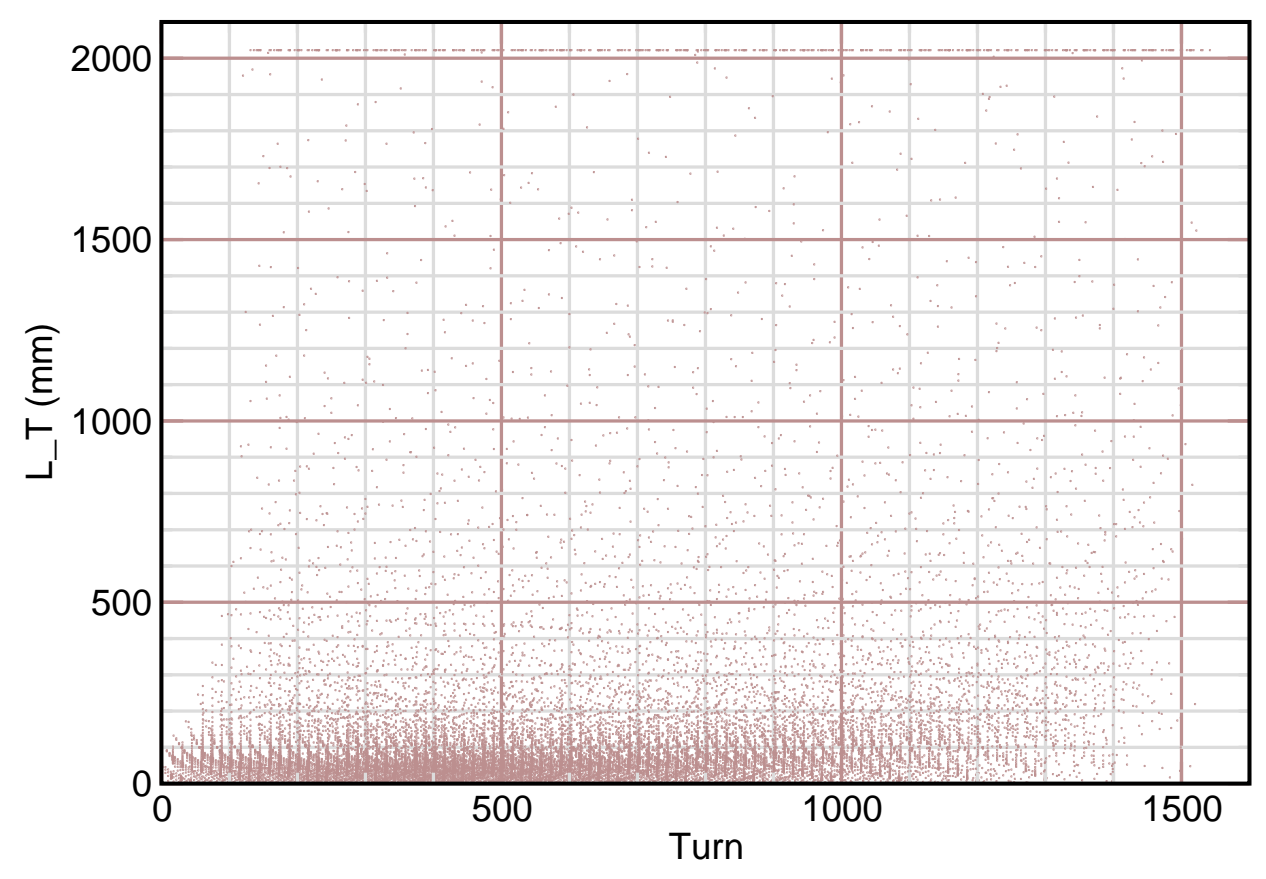

Figure 7: Scatter plot of track length $L_{T}$ and turn number for each particle's first hit on the scraper. Here the machine tune $Q=8.75$. The average $L_{T}$ is $199 \mathrm{~mm}$. Comparing with Figure $\mathbf{5}$ we see that the track lengths for this tune are considerably less. 


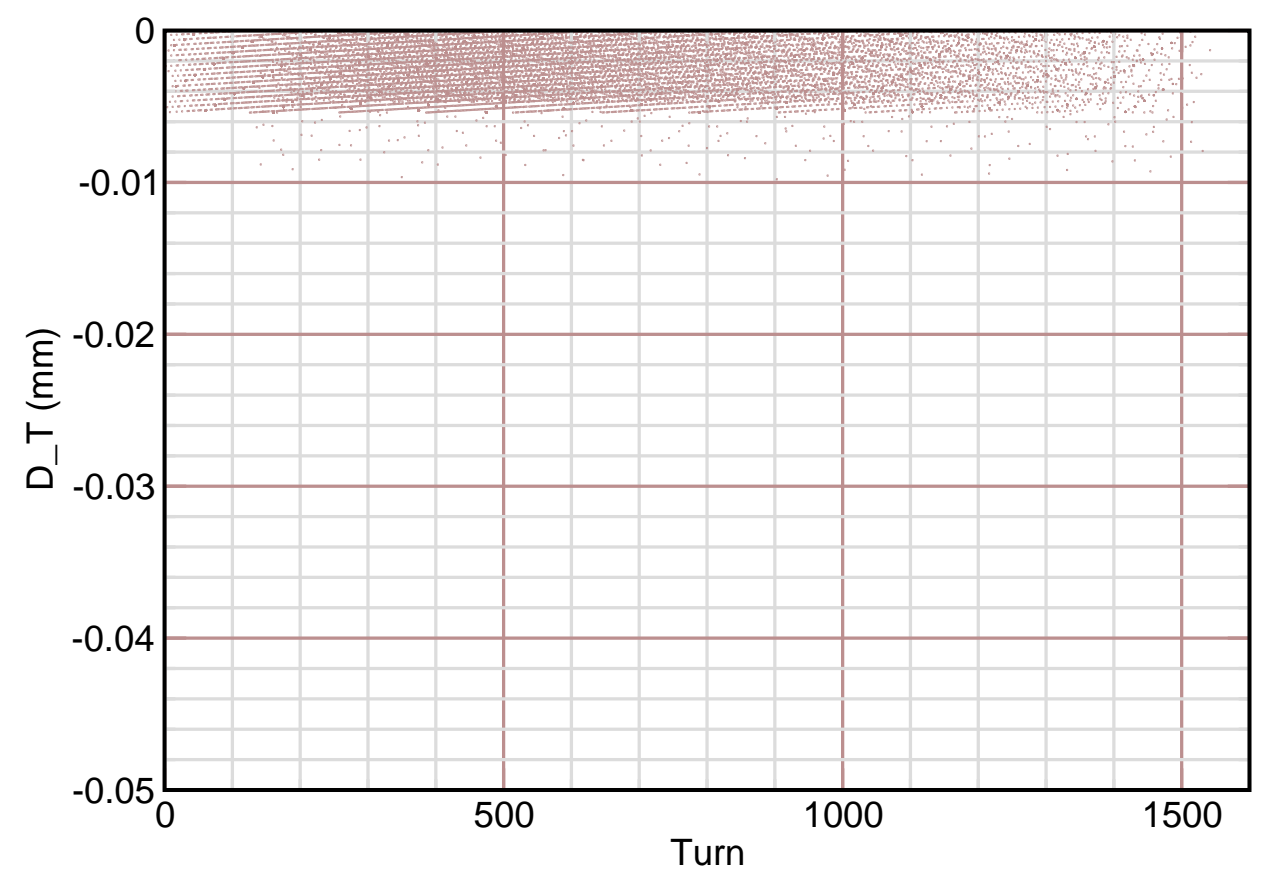

Figure 8: Scatter plot of track depth $D_{T}$ and turn number for each particle's first hit on the scraper. The machine tune $Q=8.75$. The average $D_{T}$ is $-0.00266 \mathrm{~mm}$. Comparing with Figure 6 we see that the track depths for this tune are considerably less. 


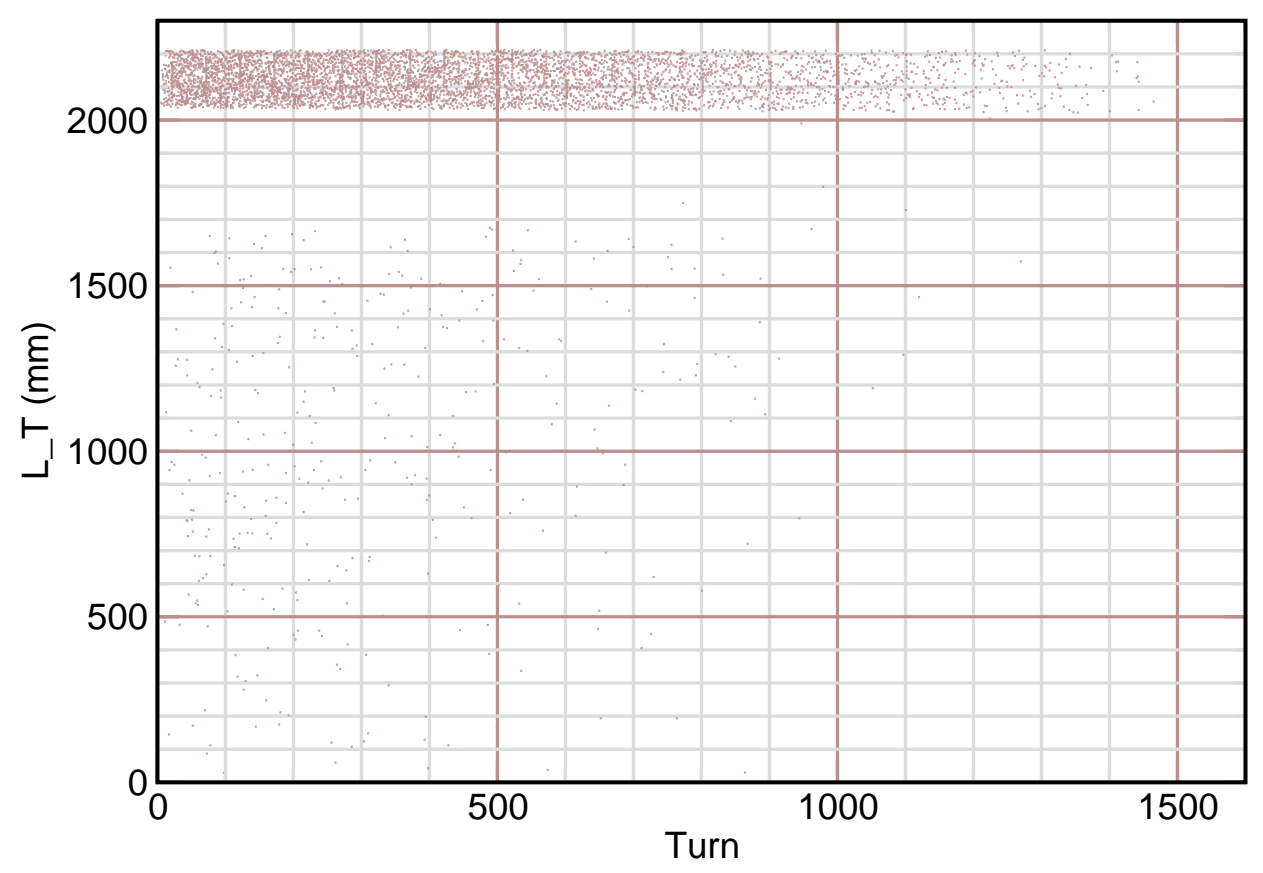

Figure 9: Scatter plot of track length $\Sigma L_{T}$ and turn number for each particle's second hit on the scraper. The machine tune $Q=8.71$. The average $\Sigma L_{T}$ is $\underline{2054 \mathrm{~mm}}$. 


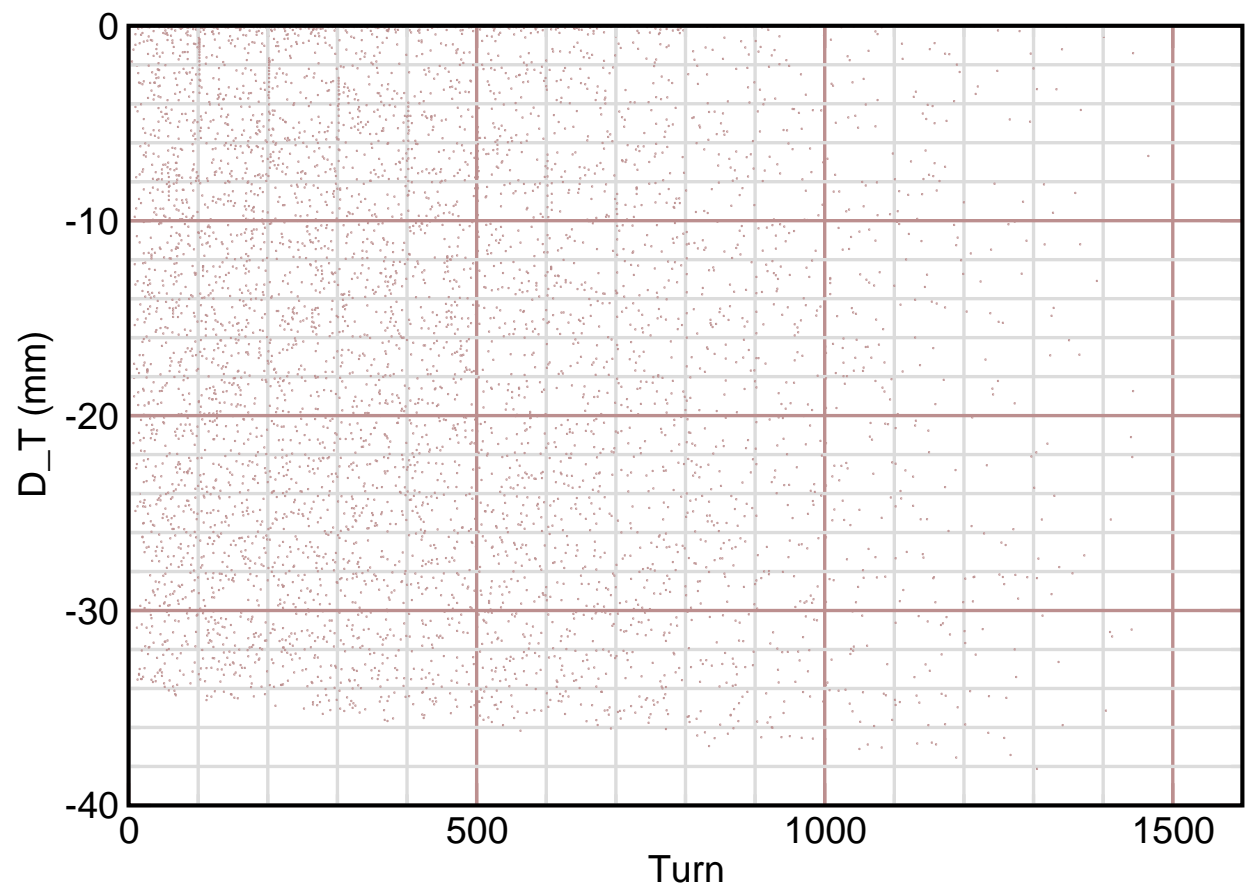

Figure 10: Scatter plot of track depth $D_{T}$ and turn number for each particle's second hit on the scraper. The machine tune $Q=8.71$. The average $D_{T}$ is $-16.1 \mathrm{~mm}$. The second-hit track depths are much larger than the first-hit depths. This is due to the $\Delta p / p$ acquired on the first hit and the nonzero dispersion in the scraper straight. 


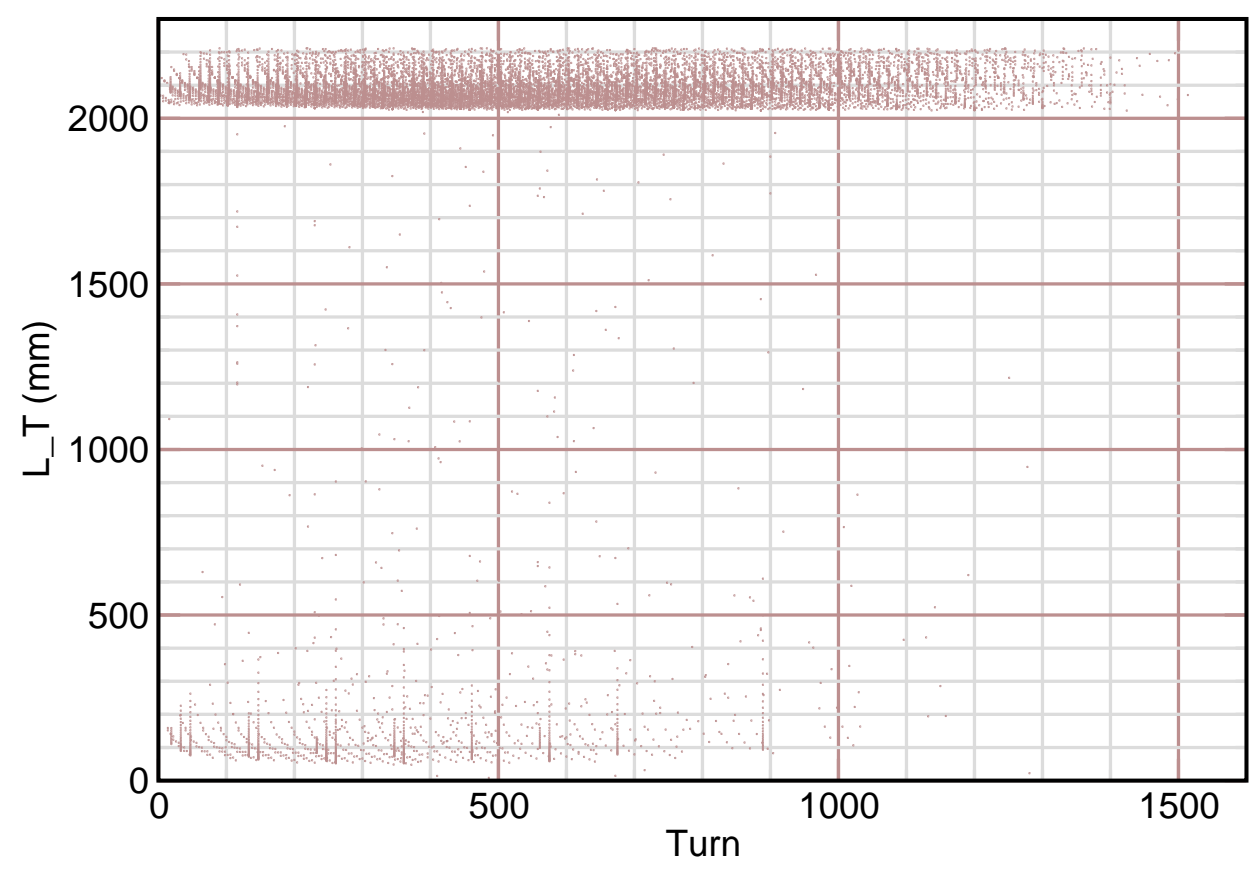

Figure 11: Scatter plot of track length $\Sigma L_{T}$ and turn number for each particle's second hit on the scraper. Here the machine tune $Q=8.75$. The average $\Sigma L_{T}$ for this tune is $1938 \mathrm{~mm}$. This is somewhat less than the value obtained from Figure 9 for tune $Q=8.71$. 


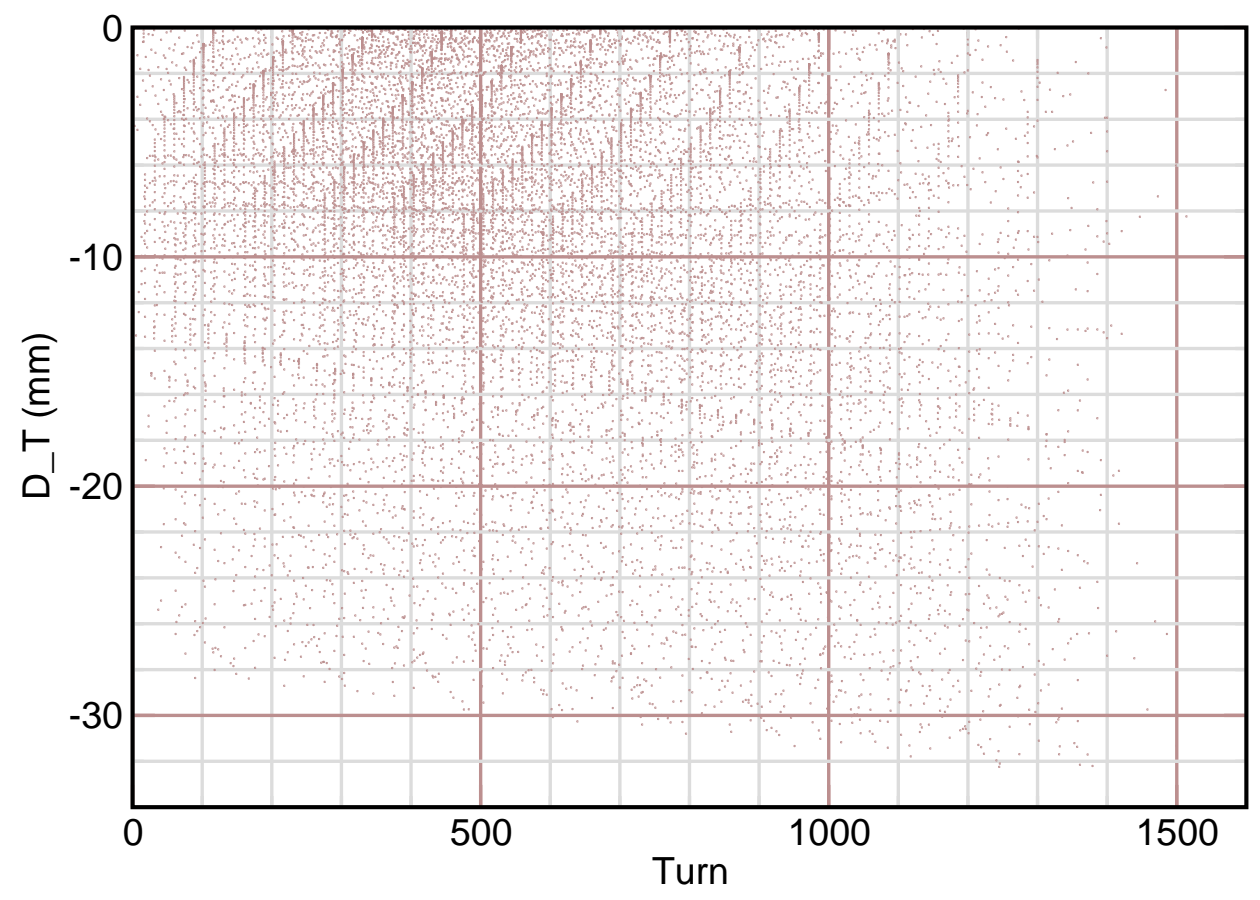

Figure 12: Scatter plot of track depth $D_{T}$ and turn number for each particle's second hit on the scraper. The machine tune $Q=8.75$. The average $D_{T}$ for this tune is $-9.02 \mathrm{~mm}$. This is somewhat less than the value obtained from Figure 10 for tune $Q=8.71$. 